\title{
A Distributed Randomized Gradient-Free Algorithm for the Non-Convex Economic Dispatch Problem
}

\author{
Jun Xie ${ }^{1, *}$, Qingyun Yu ${ }^{1}$ and Chi Cao ${ }^{2}$ (i) \\ 1 College of Energy and Electrical Engineering, Hohai University, Nanjing 211100, China; \\ yuqingyun138136@163.com \\ 2 College of Automation, Nanjing University of Posts and Telecommunications, Nanjing 210023, China; \\ sscaoch@163.com \\ * Correspondence: eejxie@163.com; Tel.: +86-25-5714-3197
}

Received: 25 November 2017; Accepted: 17 January 2018; Published: 19 January 2018

\begin{abstract}
In this paper, a distributed randomized gradient-free algorithm (DRGF) is employed to solve the complex non-convex economic dispatch problem whose non-convex constraints include valve-point loading effects, prohibited operating zones, and multiple fuel options. The DRGF uses the Gauss approximation, smoothing parameters, and a random sequence to construct distributed randomized gradient-free oracles. By employing a consensus procedure, generation units can gather local information through local communication links and then process the economic dispatch data in a distributed iteration format. Based on the principle of projection optimization, a projection operator is adopted in the DRGF to deal with the discontinuous solution space. The effectiveness of the proposed approach in addressing the non-convex economic dispatch problem is demonstrated by simulations implemented on three standard test systems.
\end{abstract}

Keywords: distributed randomized gradient-free algorithm; non-convex economic dispatch; randomized gradient-free oracles

\section{Introduction}

The general optimal economic dispatch is realized by designing unit commitment [1] for obtaining the minimum overall generation cost [2]. The centralized optimization methods are generally used in the economic dispatch problems, such as lambda iterative algorithms [3] and interior point algorithms [4], which require the objective function to be derivative. In practice, there exist conditions of valve-point loading effects in units' startup stages [5], prohibited operating zones during the operation periods [6], and choices of the several economical fuels according to the actual power outputs [5]. These conditions make the economic dispatch problem non-convex, and in this paper, we investigate a non-convex optimization algorithm to address its non-convex characteristic.

Modern metaheuristic algorithms can solve non-convex optimization problems efficiently [7], mainly including genetic algorithm (GA [5,6,8]), particle swarm optimization (PSO [9-12]), differential evolution algorithms $[13,14]$, and ant colony optimization (ACO, [15-17]). They all depend on dispatch centers to manage the information gathering from the whole system and will also use a centralized controller that will bring high communication costs. In addition, these controllers are prone to modeling errors and need a high-bandwidth communication structure with miscellaneous links [18]. Moreover, the reliability of the system is easily vulnerable to the single-point of failure [19]. Recently, the topology of power grids and communication networks are getting time-varying and extensible, but heuristic algorithms suffer a great challenge in meeting the changes.

At present, the distributed framework for economic dispatch has become a hot research topic [20-22]. Through building communication lines among the generation units and their neighbors, 
the distributed economic dispatch collects the essential iteration information and performs the real-time economic dispatch with global optimization. Simulations analyses in [20] show that the distributed dispatch will greatly cut down communication costs. The distributed gradient algorithm [21] has ensured the normal process of distributed economic dispatch when the events of units' over-limits occur. It maintains the connectivity of the communication topology after reconstructing a virtual one. In [22], with the cost function of energy storage systems non-differentiable, the sub-gradient is used as an alternative of the gradient to implement the non-convex economic dispatch. The algorithms in [20-22] are not suitable for solving non-convex optimization problems for difficulties in calculating the cost function's gradient or sub-gradient. The distributed auction-based algorithm (AA) proposed by [18] handles these non-convex constraints successfully through sharing bids among network agents and resolving the auction in a consensus procedure. However, it introduces too many intermediate variables, which will make the iteration format complex [23].

This paper adopts a distributed randomized gradient-free algorithm (DRGF) to solve non-convex economic dispatch problems with considering valve-point loading effects, prohibited operating zones, and multiple fuel options. The algorithm is based on a distributed communication structure which is cheap, reliable, and robust. Its iteration formula is implemented by employing the randomized derivative-free oracles. The effectiveness of the proposed method is verified on three test systems, and the simulation results indicate that the proposed DRGF approach can cope well with non-convex economic dispatch problems.

This paper is organized as follows. In Section 2, a non-convex economic dispatch with constraints of valve-point loading effects, prohibited operating zones, and multiple fuel options is presented. The DRGF is introduced in Section 3, and the simulation results presented in Section 4 show its effectiveness. Finally, the paper is concluded in Section 5.

\section{Non-Convex Economic Dispatch}

This paper studies an optimal loads allocation problem within a single time period. Under the premise of satisfying the system's active power balance constraint and units' power outputs limits, the total loads can be distributed among all of the units by a most economical scheme.

In conventional economic dispatch, the cost of a thermal generator unit is a quadratic function, and the dispatch center is aimed to minimize the total costs of all generation units.

$$
\min C(P)=\sum_{i=1}^{n} C_{i}\left(P_{i}\right)=\sum_{i=1}^{n}\left(a_{i} P_{i}^{2}+b_{i} P_{i}+c_{i}\right)
$$

where $C(P)$ represents the total costs of all units whose total number is $n ; P_{i}$ is the power outputs of unit $i$ and $C_{i}\left(P_{i}\right)$ is the production cost. The production characteristic coefficients are $a_{i}, b_{i}$, and $c_{i}$.

If the ramp rate limits are considered, the power outputs limits can be expressed as [10]

$$
\operatorname{Max}\left(P_{i}^{\min }, P_{i}^{o}-D R_{i}\right) \leq P_{i} \leq \operatorname{Min}\left(P_{i}^{\max }, P_{i}^{o}+U P_{i}\right)
$$

where $P_{i}^{o}$ is the previous power output, the upper and lower power output limits are $P_{i}^{\max }, P_{i}^{\min }$ respectively, and $D R_{i}$ and $U P_{i}$ are the down and up ramp limits, respectively.

The system must meet the active power balance constraints.

$$
\sum_{i=1}^{n} P_{i}=P_{d}+P_{\text {loss }}
$$

where $P_{d}$ is the total load demand. The network losses of the system are $P_{\text {loss }}$ calculated by the Kron formula [18].

$$
P_{\text {loss }}=\sum_{i=1}^{n} \sum_{j=1}^{n}\left(P_{i} B_{i j} P_{j}\right)+\sum_{i=1}^{n}\left(B_{0, i} P_{i}\right)+B_{00}
$$


where $B_{i j}, B_{0, i}$, and $B_{00}$ are the losses coefficients.

(a) Valve-point loading effects. To formulate the valve-point loading effects, a sinusoidal term is added to the original cost function [5]

$$
C_{i}\left(P_{i}\right)=a_{i} P_{i}^{2}+b_{i} P_{i}+c_{i}+\left|d_{i} \sin \left(e_{i} P_{i}-e_{i} P_{i}^{\min }\right)\right|
$$

where $d_{i}, e_{\mathrm{i}}$ are the valve-point loading coefficients of unit $i$.

(b) Prohibited operating zones. Considering this constraint can ensure the stable operation of generation units. Therefore, the units' power outputs constraints will be modified as follows [9]:

$$
\left\{\begin{array}{l}
\operatorname{Max}\left(P_{i}^{\min }, P_{i}^{o}-D R_{i}\right) \leq P_{i} \leq L_{m, i}, \quad m=1 \\
U_{m-1, i} \leq P_{i} \leq L_{m, i}, \quad m=2, \cdots M i \\
U_{m, i} \leq P_{i} \leq \operatorname{Min}\left(P_{i}^{\max }, P_{i}^{o}+U P_{i}\right), \quad m=M i
\end{array}\right.
$$

where $L_{m, i}$ and $U_{m, i}$ are the lower and upper boundaries of prohibited operating zone $m$ of unit $i$, respectively. The number of prohibited operating zones of unit $i$ is $M_{i}$.

(c) Multiple fuel options. Since units are practically provided with multiple fuel sources, each unit selects the most economical fuel in different operation range. When considering multiple fuel options, the cost function of unit $i$ with valve-point loading effects is formulated as [10]

$$
C_{i}\left(P_{i}\right)=\left\{\begin{array}{c}
a_{i}^{1} P_{i}^{2}+b_{i}^{1} P_{i}+c_{i}^{1}+\left|d_{i}^{1} \sin \left(e_{i}^{1} P_{i}-e_{i}^{1} P_{i}^{\min }\right)\right|, P_{i}^{\min } \leq P_{i} \leq P_{1 i} \\
\vdots \\
a_{i}^{q} P_{i}^{2}+b_{i}^{q} P_{i}+c_{i}^{q}+\left|d_{i}^{q} \sin \left(e_{i}^{q} P_{i}-e_{i}^{q} P_{i}^{\min }\right)\right|, P_{(q-1), i} \leq P_{i} \leq P_{i}^{\max }
\end{array}\right.
$$

where $a_{i}^{q}, b_{i}^{q}, c_{i}^{q}, d_{i}^{q}, e_{i}^{q}$ are the cost coefficients of unit $i$ under fuel $q . P_{(q-1), i}$ is the lower power outputs limit when the fuel $q$ is in use.

\section{Distributed Randomized Gradient-Free Algorithm (DRGF)}

\subsection{Treatment of Active Power Balance Constraints}

The power imbalance among economic dispatch can be described as

$$
P_{\Delta}=\sum_{i=1}^{n} P_{i}-\left(P_{d}+P_{l o s s}\right)
$$

where $P_{\Delta}$ is the power imbalance of the system, and it is the difference between the total power and the total loads plus the network active power losses. Then, each unit should adjust its power outputs to meet the supply-demand balance by keeping $P_{\Delta}$ within an acceptable level [20].

$$
P_{i} \leftarrow P_{i}+\varepsilon_{i} \cdot P_{\Delta}
$$

where $\varepsilon_{i}$ represents the power outputs adjustment factor of unit $i$ [20]. It can be calculated by $1 / n$ in this paper.

Assuming that each unit evaluates the network losses associated with its own power outputs [18] as

$$
P_{\text {loss }}(i)=P_{i} \cdot \sum_{j=1}^{n}\left(B_{i j} P_{j}\right)+P_{i} \cdot B_{0, i}+\frac{B_{00}}{n}
$$


where $P_{\text {loss }}(i)$ denotes the evaluated transmission losses by unit $i$. Based on the actual power outputs and the losses coefficients, the proposed approximation defines the power outputs including the losses, denoted by $\bar{P}_{i}$, as [18]

$$
\bar{P}_{i}=P_{i}+P_{\text {loss }}(i)
$$

where $\bar{P}_{i}$ represents the proximate power generation that contains the information of network losses. The rationale for this choice is that the losses for the currently known solution $P_{i}$ are very close to the losses for the unknown solution $\bar{P}_{i}$ [18], that is $P_{\text {loss }}(i) \approx P_{\text {loss }}^{-}(i)$.

\subsection{The Mathematical Reformulation of the Objective Function}

The non-convex economic dispatch objective Function (5) can be rewritten as:

$$
\min _{x_{i} \in X_{i}} f(x)=\sum_{i=1}^{n} f_{i}\left(x_{i}\right)
$$

where the vector $x=\left[x_{1}, x_{2}, \cdots x_{n}\right]^{T}$ represents the power output vector $P=\left[P_{1}, P_{2}, \cdots P_{n}\right]^{T} \cdot f(x)$ is the total operation cost, and $f_{i}\left(x_{i}\right)$ is the production cost of unit $i$. $X_{i}$ is the set of $x_{i}$, which represents the feasible power output space. When considering network transmission losses, $x=\left[x_{1}, x_{2}, \cdots x_{n}\right]^{T}$ refers to $\bar{P}=\left[\bar{P}_{1}, \bar{P}_{2}, \cdots \bar{P}_{n}\right]^{T}$.

Hence, the original non-convex economic dispatch problem can be rewritten as a non-convex optimization problem. Because the objective function is non-convex and the constraint conditions are discontinuous, this paper employs a distributed randomized gradient-free algorithm to address the problem. Although the objective function in the distributed scheduling model is not smooth, it satisfies the Lipschitz continuity in the variable space [23], and the smoothed version of (12) is given by

$$
\min _{x \in X} f^{\mu}(x)=\sum_{i=1}^{n} f_{i}^{\mu i}\left(x_{i}\right)
$$

where $\mu_{i}$ is the smoothing parameter of function $f_{i}^{\mu i}\left(x_{i}\right)$, which represents the Gaussian approximation of $f_{i}\left(x_{i}\right)$, explicitly given by [23]

$$
f_{i}^{\mu i}\left(x_{i}\right)=\frac{1}{\gamma} \int_{X} f_{i}\left(x_{i}+\mu_{i} \tau_{i}\right) e^{-0.5 \tau^{2}} d \tau_{i}
$$

where the constant $\gamma=(\sqrt{2 \pi})^{n}$ is a conversion factor. The random sequence $\tau_{i}$ satisfies independent distribution, according to the Gaussian distribution for each $i$. The theory of using Gauss approximation to replace the objective function is briefly shown in the Appendix A.

\subsection{The Optimization Process of the Distributed Randomized Gradient-free Algorithm}

The DRGF belongs to the distributed scheduling based on a distributed communication network structure, assuming that $n$ units communicate over a network with a time-varying topology that can be denoted by a directed graph via a nodes set $V=1,2, \cdots, n$. We define the matrix $W[k]$ as the communication network structure at the time $k$ (not negative), and the communication weight $W_{i j}[k]$ can describe the communication relationship among units. For all $i, j \in V$, the set of communication link is $E(W(k))=(i, j) \mid W_{i j}[k]>0$, and each set also includes self-edges $(i, i)$. The units' connectivity at each time can be represented by a directed graph $G(k)=(V, E(W[k]))$. For the communication matrix and units network, we make the following standard assumptions.

Assumption 1. For all $k$, the communication matrix satisfies the following properties:

(a) $W[k]$ is doubly stochastic; 
(b) The diagonal entries of $W[k]$ are positive, i.e., there exists a scalar $v>0$ such that $W_{i j}[k]>v$ for all $i \in V$. Additionally, for all $i, j \in V$, if $W_{i j}[k]>0$, then $W_{i j}[k]>v$.

Assumption 2. For all $k$, there exists a positive integer $T$ such that the graph is strongly connected.

$$
(V, E(W[k T]) \cup \cdots \cup E(W[(k+1) T-1)])
$$

Since the objective function is non-convex, it can use $\mu_{i}, f_{i}^{\mu i}\left(x_{i}\right), \tau_{i}$ to produce the random gradient-free oracles instead of gradient or sub-gradient in the iteration optimization

$$
g^{\mu i}\left(x_{i}[k]\right)=\frac{f_{i}^{\mu i}\left(x_{i}[k]+\mu_{i} \tau_{i}[k]\right)-f_{i}^{\mu i}\left(x_{i}[k]\right)}{\mu_{i}} \tau_{i}[k]
$$

where $g^{\mu i}\left(x_{i}[k]\right)$ is the random gradient-free oracle of the iteration variable $x_{i}$ at iteration $k$.

Then, the units collect neighbors' information by calculating the average weight.

$$
\overline{x_{i}}[k]=\sum_{j=1}^{n} W_{i j}[k] x_{j}[k]
$$

where $\overline{x_{i}}$ is the average weight of $x_{i}$. The unit $j$ communicates with unit $i$ in a communication weight $W_{i j}[k]$ in the $k$ th interation.

By integrating the above steps, the iteration form of optimization variables can be derived as

$$
x_{i}[k+1]=P_{X}\left[\overline{x_{i}}[k]-\alpha[k] g^{\mu i}\left(x_{i}[k]\right)\right]
$$

where $P_{X}$ is a projection operator defined in [24]. Iteration step-sizes satisfy the following conditions:

$$
\alpha[k]>0, \lim _{k \rightarrow \infty} \sum \alpha[k]=\infty, \lim _{k \rightarrow \infty} \sum(\alpha[k])^{2}<\infty
$$

According to Formulas (13)-(19), the flowchart of the DRGF can be given by Figure 1. Further, the implementation process of the non-convex economic dispatch via the DRGF can be designed, as shown in Figure 2.

The specific procedures of Figure 2 are as follows:

(1) The input data of operation parameters include the production characteristic coefficients $\left(a_{i}, b_{i}\right.$, $c_{i}, d_{i}$, and $\left.e_{i}\right)$, total number of generation units $(n)$, the total load demand $\left(P_{d}\right)$, and the losses coefficients $\left(B_{i j}, B_{0, i}\right.$, and $\left.B_{00}\right)$. The operation constraints contain the ramp rate limits, the lower and upper boundaries of prohibited operating zones, and the operation ranges of different fuel options.

(2) The initial power outputs may meet the initial supply-demand balance in Formula (3). So, all of them can be set to the same value $\frac{P_{d}}{n}$ and $P_{d}$ is given in references $[5,9,25]$.

(3) After setting up the initialization power output, the $P_{\text {loss }}(i)$ will be calculated by Formula (10) for considering the network losses. Then, by Formula (11), it can get the proposed approximation $\bar{P}_{i}$ which contains the information of network losses.

(4) When the power output falls into the prohibited operating zones, shown in Formula (6), then the unit will operate at its upper boundary (if $P_{i}[k+1]<P_{i}[k]$ ) or lower boundary (if $P_{i}[k+1]>P_{i}[k]$ ).

(5) According to the actual power output, the units select the most economical fuel $q$ with the corresponding cost coefficients $a_{i}^{q}, b_{i}^{q}, c_{i}^{q}, d_{i}^{q}, e_{i}^{q}$ and its operation curve follows the Formula (7).

(6) If the valve-point loading effects are considered, the sinusoidal term with coefficients $d_{i}, e_{i}$ will be added to units' cost functions. 
(7) When all the equality and inequality constraints are satisfied, it will form the objective functions and feasible solution space, such as shown in Formula (12).

(8) Next, implement the DRGF optimization (shown in Figure 1) and perform the $k$ th optimization.

(9) Re-examine the power outputs constraints, and if the constraints meet the Formula (6), it will get into the next step; otherwise, it will go back to step (3).

(10) Calculate the power imbalance $P_{\Delta}$, and if it exceeds the allowable value, a step of adjustment will be implemented, which is $P_{i} \leftarrow P_{i}+\varepsilon_{i} \cdot P_{\Delta}$, then the optimization process will restart from step (3) until the power imbalance $P_{\Delta}$ less than $0.01 \mathrm{MW}$, and finally, the optimum solution can be obtained.

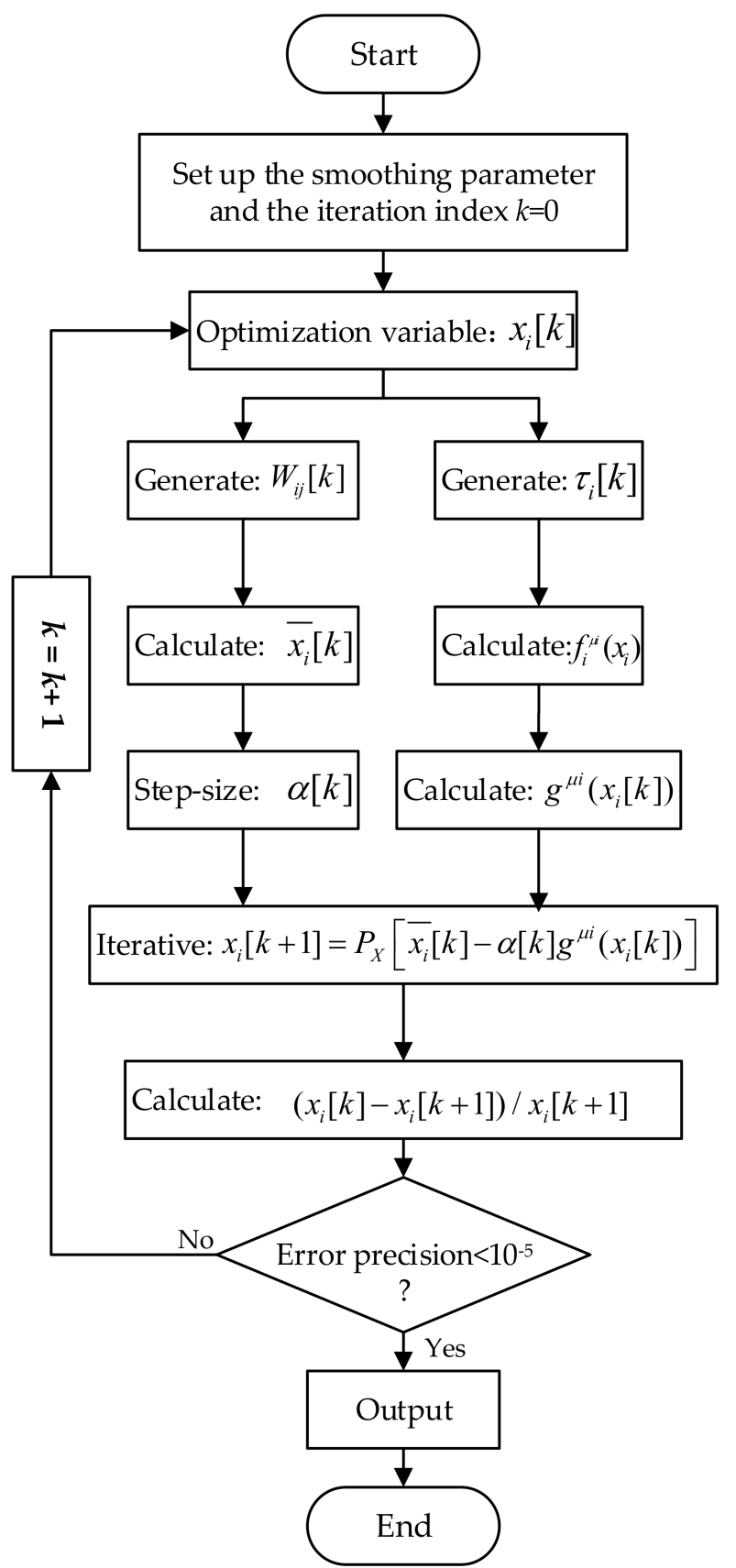

Figure 1. The flowchart of the distributed randomized gradient-free algorithm (DRGF). 


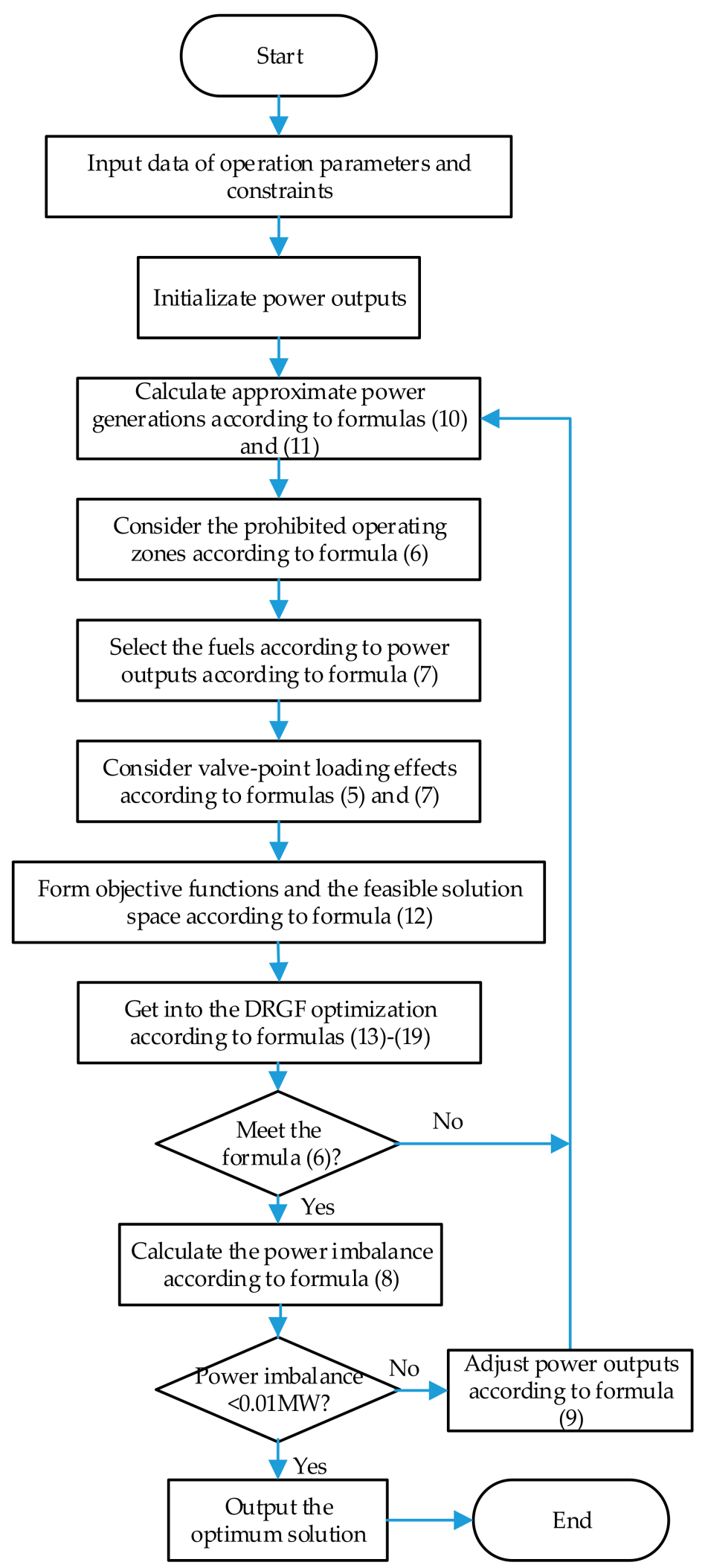

Figure 2. The non-convex economic dispatch process via the DRFG. 
The pseudo code of the distributed randomized gradient-free algorithm for non-convex economic dispatch problem (Algorithm 1) is shown as follows:

\footnotetext{
Algorithm 1: the distributed randomized gradient-free algorithm for non-convex economic dispatch problem
}

1. INPUT: The operation parameters and constraints and the initial power outputs $P_{i}[0]$.

2. OUTPUT: The optimal power outputs

3. PROCEDURE:

4. for $i=1$ to $n$ do

5. Calculate the network losses by Formula (10)

$$
P_{\text {loss }}(i)=P_{i} \cdot \sum_{j=1}^{n}\left(B_{i j} P_{j}\right)+P_{i} \cdot B_{0, i}+\frac{B_{00}}{n}
$$

6. Calculate approximate power generations by Formula (11):

$$
\bar{P}_{i}=P_{i}+P_{\text {loss }}(i)
$$

7. Deal with the prohibited operating zones according to Formula (6):

8. When $P_{i}[k+1]<U_{m, i} \& \& P_{i}[k+1]<L_{m, i}$ do

9. $\quad$ if $P_{i}[k+1]<P_{i}[k]$ then $P_{i}[k+1]=U_{m, i}$

10. else if $P_{i}[k+1]>P_{i}[k]$ then $P_{i}[k+1]<L_{m, i}$

11. end if

12. Consider valve-point loading effects according to Formula (5):

$$
C_{i}\left(P_{i}\right)=a_{i} P_{i}^{2}+b_{i} P_{i}+c_{i}+\left|d_{i} \sin \left(e_{i} P_{i}-e_{i} P_{i}^{\min }\right)\right|
$$

13. Select the fuels according to power outputs according to Formula (7):

$$
C_{i}\left(P_{i}\right)=\left\{\begin{array}{c}
a_{i}^{1} P_{i}^{2}+b_{i}^{1} P_{i}+c_{i}^{1}+\left|d_{i}^{1} \sin \left(e_{i}^{1} P_{i}-e_{i}^{1} P_{i}^{\min }\right)\right|, P_{i}^{\min } \leq P_{i} \leq P_{1 i} \\
\vdots \\
a_{i}^{q} P_{i}^{2}+b_{i}^{q} P_{i}+c_{i}^{q}+\left|d_{i}^{q} \sin \left(e_{i}^{q} P_{i}-e_{i}^{q} P_{i}^{\min }\right)\right|, P_{(q-1), i} \leq P_{i} \leq P_{i}^{\max }
\end{array}\right.
$$

14. end for

15. Optimize sub-problem1:

$$
\begin{aligned}
& \min _{x \in X} f^{\mu}(x)=\sum_{i=1}^{n} f_{i}^{\mu i}\left(x_{i}\right) \\
& \text { where } \\
& f_{i}^{\mu i}\left(x_{i}\right)=\frac{1}{\gamma} \int_{X} f_{i}\left(x_{i}+\mu_{i} \tau_{i}\right) e^{-0.5 \tau^{2}} d \tau_{i}
\end{aligned}
$$

16. Generate $W_{i j}[k]$ and $\tau_{i}[k]$; Calculate $\overline{x_{i}}, \alpha[k]$, and $g^{\mu i}\left(x_{i}[k]\right)$.

17. Go into the optimal iteration process:

18. for $\mathrm{k}=0$ to $\mathrm{K}$ do

$$
x_{i}[k+1]=P_{X}\left[\overline{x_{i}}[k]-\alpha[k] g^{\mu i}\left(x_{i}[k]\right)\right]
$$

19. Calculate the error precision

20. if the error precision less than $10^{-5}$ then output theoptimization

21. else reiterate the step 16

22. end if

23. Revalidate the constraint conditions in Formulas (2) and (6)

24. Calculate the power imbalance by Formula (8)

$$
P_{\Delta}=\sum_{i=1}^{n} P_{i}-\left(P_{d}+P_{\text {loss }}\right)
$$

25. if $P_{\Delta} \leq 0.01$ then output the optimum solution

26. $\quad$ else do $P_{i} \leftarrow P_{i}+\varepsilon_{i} \cdot P_{\Delta}$ and then return to step 4 .

27. end if

28. end for

\section{Numerical Examples}

Three test systems are employed for simulation to verify the effectiveness of the proposed DRGF in solving non-convex economic scheduling problems. The solution space in this paper is compared with the ones get by existing methods. 


\subsection{Description of the Test Systems}

The first test system consists of six units whose cost functions are all quadratic, with prohibited operating zones for four units and network losses. The total load demand is $1263 \mathrm{MW}$, and the details of the six units are reported in [9].

The second system consists of 10 units with considering both valve-point loading effects and multiple fuel options. The unit- 1 has two fuel options, while others have three. The total load demand is $2700 \mathrm{MW}$, and the details of the ten units are shown in [5].

The third test system contains 40 units with cost functions modeling valve-point loading effects. The load demand is 10,500 MW, and the details of the 40 units are given in [25].

\subsection{Setting of Simulation}

The DRGF iterates over the power outputs based on the randomized derivative-free oracles. According to the flow chart, the parameters of $\mu_{i}$ and $\alpha[k]$ may affect the convergence of the algorithm. In [26], the influence of the number of optimization variables and $\mu_{i}$ on the convergence of the algorithm is studied by several examples. It is a fact that, with the number of optimization variables increasing, the DRGF algorithm requires more iteration to achieve optimal result. $\mu_{i}$ is set at 0.0005 in the test examples. A dynamic iteration step-size is put to use in this paper, such as $\alpha[k]=1 / \sqrt{k+1}$. The coding is written with MATLAB 2012 programming language and executed on the Intel (R) Core (TM) i5, 2.20-GHz, 8.0-GB RAM processor.

\subsection{Solution Quality}

Tables 1-3 provides the optimization results of six units, ten units and forty units test systems by using the DRGF algorithm, including the optimal power outputs, the total power outputs (TP), the total network losses (TL), and the total costs (TC). Figures 3-5 show the optimization curves of TC by various algorithms.

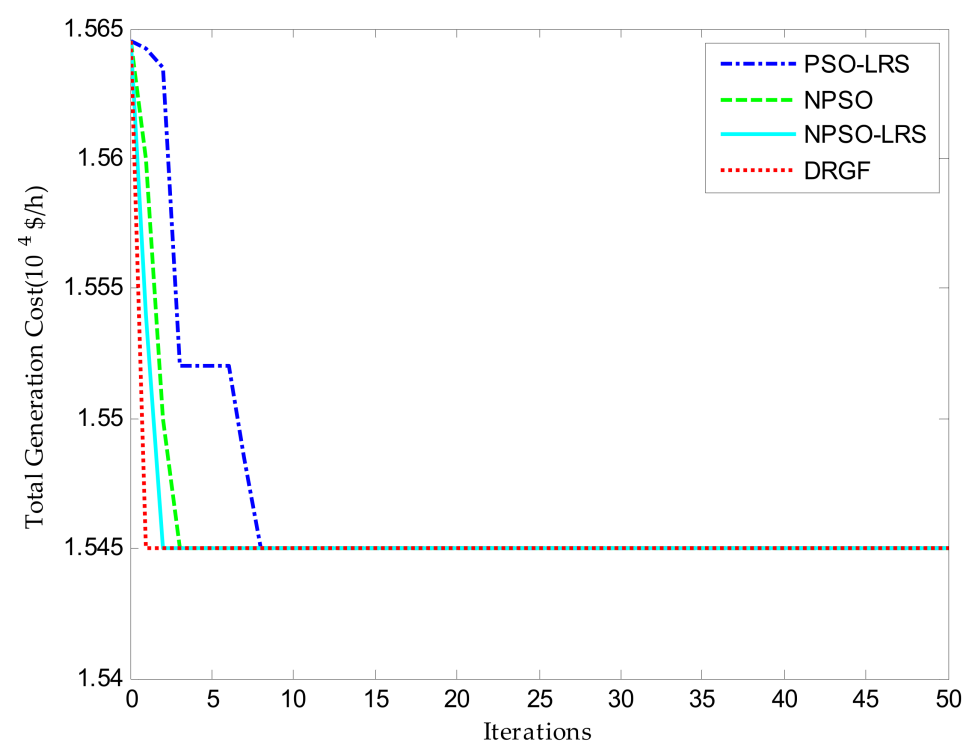

Figure 3. Optimization curves of six units test system. PSO-LRS: particle swarm optimization-simple local random search; NPSO: new particle swarm optimization; NPSO-LRS: new particle swarm optimization- simple local random search. 


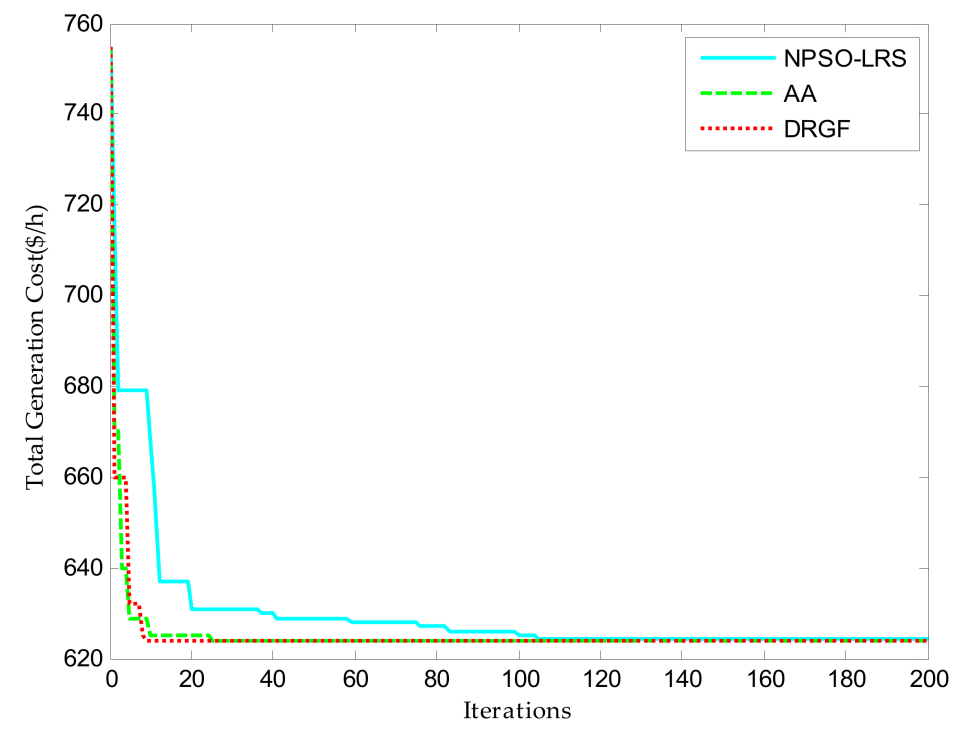

Figure 4. Optimization curves of 10 units test system. AA: auction-based algorithm.

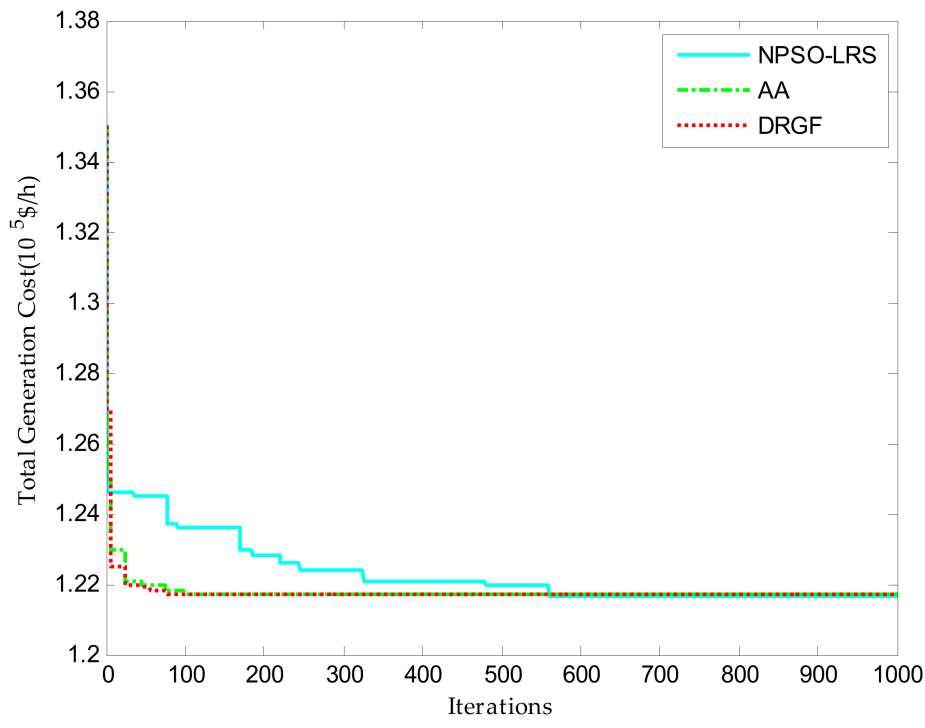

Figure 5. Optimization curves of 40 units test system.

Table 1. Optimization results of six units test system. PSO-LRS: particle swarm optimization-simple local random search; NPSO: new particle swarm optimization; NPSO-LRS: new particle swarm optimization- simple local random search. DRGF: distributed randomized gradient-free algorithm.

\begin{tabular}{ccccc}
\hline \multirow{2}{*}{ The Units } & \multicolumn{4}{c}{ Power Outputs by Different Algorithms (MW) } \\
\cline { 2 - 5 } & PSO-LRS & NPSO & NPSO-LRS & DRGF \\
\hline P1 & 447.4440 & 447.4734 & 446.9600 & 447.4859 \\
P2 & 173.3430 & 173.1012 & 173.3944 & 173.3303 \\
P3 & 263.3646 & 262.6804 & 263.3436 & 263.3798 \\
P4 & 139.1279 & 139.4156 & 139.5120 & 139.1003 \\
P5 & 165.5076 & 165.3002 & 164.7089 & 165.4978 \\
P6 & 87.16980 & 87.97610 & 89.01620 & 87.13306 \\
TP & 1275.95 & 1275.95 & 1275.94 & 1275.95 \\
TL & 12.9571 & 12.9470 & 12.9361 & 12.9584 \\
TC & 15,450 & 15,450 & 15,450 & 15,450 \\
\hline
\end{tabular}


Table 2. Optimization results of 10 units test system.

\begin{tabular}{|c|c|c|c|c|c|c|}
\hline \multirow{3}{*}{ The Units } & \multicolumn{6}{|c|}{ Power Outputs by Different Algorithms (MW) } \\
\hline & \multicolumn{2}{|c|}{ NPSO-LRS } & \multicolumn{2}{|c|}{ Auction-Based Algorithm (AA) } & \multicolumn{2}{|c|}{ DRGF } \\
\hline & Fuels & Results & Fuels & Results & Fuels & Results \\
\hline P1 & 2 & 223.3352 & 2 & 219.1314 & 2 & 219.1312 \\
\hline P2 & 1 & 212.1957 & 1 & 212.6569 & 1 & 212.6579 \\
\hline P3 & 1 & 276.2167 & 1 & 278.6388 & 1 & 278.6388 \\
\hline P4 & 3 & 239.4187 & 3 & 237.4048 & 3 & 237.4046 \\
\hline P5 & 1 & 274.6470 & 3 & 279.9248 & 3 & 279.9239 \\
\hline P6 & 3 & 239.7974 & 1 & 238.9899 & 1 & 238.9899 \\
\hline P7 & 1 & 285.5388 & 3 & 290.0868 & 3 & 290.0877 \\
\hline P8 & 3 & 240.6323 & 3 & 237.4048 & 3 & 237.4060 \\
\hline P9 & 3 & 429.2637 & 3 & 429.9043 & 3 & 429.9043 \\
\hline P10 & 1 & 278.9541 & 1 & 275.8573 & 1 & 275.8575 \\
\hline $\mathrm{TP}(\$ / \mathrm{h})$ & \multicolumn{2}{|c|}{2700.0} & \multicolumn{2}{|c|}{2700.0} & \multicolumn{2}{|c|}{2700.0} \\
\hline $\mathrm{TC}(\$ / \mathrm{h})$ & \multicolumn{2}{|c|}{624.13} & \multicolumn{2}{|c|}{623.95} & \multicolumn{2}{|c|}{623.95} \\
\hline
\end{tabular}

Table 3. Optimization results of 40 units test system.

\begin{tabular}{cccccc}
\hline Units & Results (MW) & Units & Results (MW) & Units & Results (MW) \\
\hline P1 & 110.9751 & P15 & 394.5920 & P29 & 10.8977 \\
P2 & 110.9751 & P16 & 394.5920 & P30 & 88.1956 \\
P3 & 97.88472 & P17 & 489.5454 & P31 & 189.1586 \\
P4 & 180.0803 & P18 & 489.5454 & P32 & 159.7586 \\
P5 & 88.1956 & P19 & 511.7249 & P33 & 189.1586 \\
P6 & 106.1117 & P20 & 511.7249 & P34 & 164.8803 \\
P7 & 260.2433 & P21 & 523.4980 & P35 & 164.8803 \\
P8 & 285.1205 & P22 & 523.4980 & P36 & 164.8803 \\
P9 & 285.1205 & P23 & 523.4980 & P37 & 96.6075 \\
P10 & 204.9651 & P24 & 523.4980 & P38 & 109.2075 \\
P11 & 168.9643 & P25 & 523.4980 & P39 & 109.2075 \\
P12 & 168.9643 & P26 & 523.4980 & P40 & 511.7249 \\
P13 & 215.0420 & P27 & 10.8977 & TP & 10500 \\
P14 & 304.2920 & P28 & 10.8977 & TC & $121,788.7$ \\
\hline
\end{tabular}

A varieties of centralized algorithms have been successful presented to solve the six units test system and three improved PSO algorithms have achieved the best result with a minimum cost of $15,450 \$ / \mathrm{h}$. The new particle swarm optimization (NPSO) [10] and new particle swarm optimizationsimple local random search (NPSO-LRS) [10] are almost the same in convergence rate and show their superiority over the particle swarm optimization-simple local random search (PSO-LRS) algorithm. The NPSO algorithm performs well due to the extra diversification provided by the worst experience component. As shown in Figure 3, the DRGF algorithm can also realize the same minimum generation cost. And it is slightly better than NPSO-LRS due to the distributed searching ability.

The 10 units test system mainly considers valve-point loading effects and multiple fuel options. As shown in Figure 4, a varieties of centralized algorithms have been successful presented to solve the 10 units test system, and three improved PSO algorithms have achieved the best result with a minimum cost of $624.13 \$ / \mathrm{h}$. Here, DRGF is faster in convergence rate than that of PSO algorithm. The behavior of AA falls between those of DRGF and NPSO-LRS. For all the three test cases, DRGF runs first in the performance ladder. The proposed worst experience component in the velocity update equation, and the random gradient-free search procedure strengthens the DRGF. From Figure 4, it can be found that the DRGF algorithm can achieve the optimal solution (about $623.95 \$ / \mathrm{h}$ ) as the AA algorithm does, which indicates that the distributed algorithm DRGF is superior to the NPSO-LRS.

When only considering valve-point loading effects, the 40 units test system can be optimized by the DRGF algorithm whose optimization results are provided in Table 3. As a comparison, Table 4 presents the optimal cost under different scheduling methods, and Figure 5 shows the cost curves of the NPSO-LRS, DRGF, and AA. It can be seen that the two distributed economic dispatch schemes 
(DRGF and AA) obtain a cost of 121,788.70 \$/h within 100 iterations, and NPSO-LRS gets a cost of $121,664.43 \$ / \mathrm{h}$, the minimum in 100 trials, at about the 570th iteration. In terms of costs, the NPSO-LRS is slightly better than the distributed methods DRGF and AA. But it is found that the convergence speed of the distributed algorithm is obviously better than the centralized algorithm. The DRGF approach is simple and adaptive to large-scale generation systems.

Table 4. Costs for 40 units test system from 100 trials.

\begin{tabular}{ccccc}
\hline \multirow{2}{*}{ Algorithms } & \multirow{2}{*}{ Dispatch Types } & \multicolumn{3}{c}{ TC $\mathbf{( \$ \mathbf { h } )}$} \\
\cline { 3 - 5 } & & Minimum & Maximum & Average \\
\hline GA (Genetic Algorithm) & Centr. & $135,070.00$ & $137,980.00$ & $137,760.00$ \\
\hline PSO & Centr. & $130,380.00$ & $137,740.00$ & $134,970.00$ \\
\hline NPSO & Centr. & $121,704.74$ & $122,995.10$ & $122,221.37$ \\
\hline PSO-LRS & Centr. & $122,035.79$ & $123,461.68$ & $122,558.46$ \\
\hline NPSO-LRS & Centr. & $121,664.43$ & $122,981.59$ & $122,209.32$ \\
\hline AA & Distr. & & $121,788.70$ \\
\hline DRGF & Distr. & & $121,788.70$ \\
\hline
\end{tabular}

\subsection{Robustness Test of the Algorithm}

Due to the randomness of the calculation process, results acquired from intelligent optimization algorithms are not unique. A great deal of optimization trials, e.g., 100 trials, should be conducted to examine their performance and the optimal operation cost is listed in Table 4 including the minimum, the maximum, and the average data. To compare the results in a statistical manner, the frequencies of attainment of a cost within the specific ranges are presented in Table 5.

Table 5. Frequency of convergence for 40 units test system from 100 trials.

\begin{tabular}{cccc}
\hline \multirow{2}{*}{ Algorithms } & \multicolumn{3}{c}{ Range of Costs $(\mathbf{1 0} \mathbf{5} \mathbf{\$} \mathbf{h})$} \\
\cline { 2 - 4 } & $\mathbf{1 . 2 1 5}-\mathbf{1 . 2 2 0}$ & $\mathbf{1 . 2 2 0 - 1 . 2 2 5}$ & $\mathbf{1 . 2 2 5 - 1 . 2 3 0}$ \\
\hline NPSO-LRS & 16 & 61 & 23 \\
AA & 100 & 0 & 0 \\
DRGF & 100 & 0 & 0 \\
\hline
\end{tabular}

As shown in Table 4, the maximum and average cost of centralized algorithms have a large difference with the minimum costs, such as PSO for $7360 \$ / \mathrm{h}$ and 4590 \$/h; NPSO-LRS has achieved better optimization results in many algorithms, with the difference of $1317.16 \$ / \mathrm{h}$ and $544.89 \$ / \mathrm{h}$, respectively. In contrast, the optimal solutions of the distributed scheduling are fixed (seen in Table 5), which demonstrate that the robustness of distributed algorithm is far better than the centralized algorithms. As a result, the DRGF algorithm is more suitable for solving complex non-convex economic dispatch problems.

In this paper, the average CPU (Central Processing Unit) running time is used to analyze the computation efficiency of the DRGF algorithm. We examine the average optimization time $T_{o}$ and the operation time $T_{f}$ with the fixed iteration number (200 steps) to achieve the optimal results under each test system, respectively. The algorithms run 100 times on the MATLAB 2012a, and the average results are shown in Table 6.

Table 6. The time of $T_{o}$ and $T_{f}$ by adopting different algorithms.

\begin{tabular}{ccccc}
\hline \multirow{2}{*}{ Algorithms } & \multicolumn{2}{c}{$T_{f}$ (Second) } & \multicolumn{2}{c}{$T_{\boldsymbol{o}}$ (Second) } \\
\cline { 2 - 5 } & $\mathbf{1 0}$ Units & 40 Units & 10 Units & 40 Units \\
\hline NPSO-LRS & 0.00472 & 0.01193 & 0.00519 & 0.05639 \\
AA & 0.00118 & 0.00242 & 0.00146 & 0.00786 \\
DRGF & 0.00113 & 0.00229 & 0.00132 & 0.00723 \\
\hline
\end{tabular}


As is known from the above figure, the algorithm NPSO-LRS will take more time to get the optimal results when the number of units is lager. By analysis and comparison with NPSO-LRS, it can be seen that the distributed scheduling schemes (AA and DRGF) for optimizing non-convex scheduling need less time. Due to different algorithms frameworks, centralized dispatches need to collect all the information of the optimization variables and then process and transmit them in a centralized way. The computational efficiency of the distributed DRGF algorithm is better than NPSO-LRS and AA algorithm.

\section{Conclusions}

This paper establishes a non-convex economic dispatch model after considering the constraints of valve-point loading effects, prohibited operating zones, and multiple fuel options. According to these complex non-convex characteristics, this paper introduces a DRGF with randomized derivative-free oracles instead of sub-gradients to solve the economic dispatch model. Simulation scenes are implemented on three test systems, and optimization results obtained by different algorithms have been made further comparisons. The analysis validates that the DRGF algorithm can effectively address the complex economic dispatch problem with valve-point loading effects, prohibited operating zones and multiple fuel options. In the next study, effort should be spent on improving the iteration efficiency of the proposed algorithm.

Acknowledgments: This study is supported by the Major State Basic Research Development Program of China (Grant No. 2016YFB0901100) and the National Science Foundation of China (Grant No. 51577051).

Author Contributions: All the authors contributed to this work. Jun XIE provided critical guidance to this research and checked the overall logic of this work. Qingyun YU contributed toward the modeling framework. Chi CAO designed the study, developed the mathematical model, and performed the analysis and simulations.

Conflicts of Interest: The authors declare no conflict of interest.

\section{Appendix A}

According to [20] (Theorems 1 and 4, Formulas (11) and (A3)), the following lemma is introduced to provide some important properties of the function $f_{i}^{\mu i}\left(x_{i}\right)$ and the random gradient-free oracle $g^{\mu i}\left(x_{i}[k]\right)$.

Lemma A1. for each $i$, there has

(a) $f_{i}^{\mu i}\left(x_{i}\right)$ is convex and differentiable, and it satisfies

$$
f_{i}\left(x_{i}\right) \leq f_{i}^{\mu i}\left(x_{i}\right) \leq f_{i}\left(x_{i}\right)+\sqrt{n} \mu_{i} G_{0}\left(f_{i}\right)
$$

(b) The gradient $\nabla f_{i}^{\mu i}\left(x_{i}\right)$ satisfies

$$
\left[g^{\mu i}\left(x_{i}[k]\right)\right]=\nabla f_{i}^{\mu i}\left(x_{i}\right)
$$

(c) The random gradient-free oracle $g^{\mu i}\left(x_{i}[k]\right)$ satisfies

$$
\left[\left\|g^{\mu i}\left(x_{i}[k]\right)\right\|^{2}\right] \leq(n+4)^{2} G_{0}\left(f_{i}\right)^{2}
$$

where $f_{i}$ is Lipschitz continuous over $x$ with Lipschitz constant $G_{0}\left(f_{i}\right)$. $E[x]$ denotes the expected value of a random variable $x$. The further principle description and proof can be found in [26]. 


\section{References}

1. Tahanan, M.; Ackooij, W.V.; Frangioni, A.; Lacalandra, F. Large scale Unit commitment under uncertainty. Q. J. Oper. Res. 2015, 13, 115-171.

2. Yang, Z.Q.; Xiang, J.; Li, Y.J. Distributed consensus based supply-demand balance algorithm for economic dispatch problem in a smart grid with switching graph. IEEE Trans. Ind. Electr. 2017, 64, 1600-1610. [CrossRef]

3. Lin, C.E.; Viviani, G.L. Hierarchical economic dispatch for piecewise quadratic cost functions. IEEE Trans. Power Appar. Syst. 1984, 103, 1170-1175. [CrossRef]

4. Lin, W.M.; Chen, S.J. Bid-based dynamic economic dispatch with an efficient interior point algorithm. Int. J. Electr. Power Energy Syst. 2002, 24, 51-57. [CrossRef]

5. Chiang, C.L. Improved genetic algorithm for power economic dispatch of units with valve-point effects and multiple fuels. IEEE Trans. Power Syst. 2005, 20, 1690-1699. [CrossRef]

6. Amjady, N.; Rad, H.N. Nonconvex economic dispatch with ac constraints by a new real coded genetic algorithm. IEEE Trans. Power Syst. 2009, 24, 1489-1502. [CrossRef]

7. Blum, C.; Aguilera, M.J.B.; Roli, A.; Sampels, M. An Emerging Approach to Optimization; Springer: Berlin, Germany, 2008.

8. Amjady, N.; Rad, H.N. Economic dispatch using an efficient real-coded genetic algorithm. IET Gener. Transm. Distrib. 2009, 3, 266-278. [CrossRef]

9. Gaing, Z.L. Particle swarm optimization to solving the economic dispatch considering the generator constraints. IEEE Trans. Power Syst. 2003, 18, 1187-1195. [CrossRef]

10. Selvakumar, A.I.; Thanushkodi, K. A new particle swarm optimization solution to nonconvex economic dispatch problems. IEEE Trans. Power Syst. 2007, 22, 42-51. [CrossRef]

11. Chaturvedi, K.T.; Pandit, M.; Srivastava, L. Self-organizing hierarchical particle swarm optimization for non-convex economic dispatch. IEEE Trans. Power Syst. 2008, 23, 1079-1087. [CrossRef]

12. Panigrahi, B.K.; Pandi, V.R.; Das, S. Adaptive particle swarm optimization approach for static and dynamic economic load dispatch. Energy Convers. Manag. 2008, 49, 1407-1415. [CrossRef]

13. Bhattacharya, A.; Chattopadhyay, P.K. Hybrid differential evolution with biogeography based optimization for solution of economic load dispatch. IEEE Trans. Power Syst. 2010, 25, 1955-1964. [CrossRef]

14. Nomana, N.; Iba, H. Differential evolution for economic load dispatch problems. Electr. Power Syst. Res. 2008, 78, 1322-1331. [CrossRef]

15. D'Andreagiovanni, F.; Krolikowski, J.; Pulaj, J. A fast hybrid primal heuristic for multiband robust capacitated network design with multiple time periods. Appl. Soft Comput. 2015, 26, 497-507. [CrossRef]

16. Pothiya, S.; Ngamroo, I.; Kongprawechnon, W. Antcolony optimisation for economic dispatch problem with non-smooth cost functions. Int. J. Electr. Power Energy Syst. 2010, 32, 478-487. [CrossRef]

17. Schlüter, M.; Egea, J.A.; Banga, J.R. Extended ant colony optimization for non-convex mixed integer nonlinear programming. Comput. Oper. Res. 2009, 36, 2217-2229. [CrossRef]

18. Binetti, G.; Davoudi, A.; Naso, D.; Turchiano, B.; Lewis, F.L. A distributed auction-based algorithm for the non-convex economic dispatch problem. IEEE Trans. Ind. Inf. 2014, 10, 1124-1132. [CrossRef]

19. Yang, Q.; Barria, J.A.; Green, T.C. Communication infrastructures for distributed control of power distribution networks. IEEE Trans. Ind. Inf. 2011, 7, 316-327. [CrossRef]

20. Rahbari-Asr, N.; Unnati, O.; Zhang, Z.; Chow, M.-Y. Incremental welfare consensus algorithm for cooperative distributed generation/demand response in smart grid. IEEE Trans. Smart Grid 2014, 5, 2836-2845. [CrossRef]

21. Zhang, W.; Liu, W.; Wang, X. Online optimal generation control based on constrained distributed gradient algorithm. IEEE Trans. Power Syst. 2015, 30, 35-45. [CrossRef]

22. Yang, H.M.; Yi, D.X.; Zhao, J.H.; Dong, Z.Y. Distributed optimal dispatch of virtual power plant via limited communication. IEEE Trans. Power Syst. 2013, 28, 3511-3512. [CrossRef]

23. Nesterov, Y.; Spokoiny, V. Random Gradient-Free Minimization of Convex Functions. Found. Comput. Math. 2017, 17, 527-566. [CrossRef]

24. Nedic, A.; Ozdaglar, A.; Parril, P.A. Constrained consensus and optimization in multi-agent networks. IEEE Trans. Autom. Control 2010, 55, 922-938. [CrossRef] 
25. Sinha, N.; Chakrabarti, R.; Chattopadhyay, P.K. Evolutionary programming techniques for economic load dispatch. IEEE Trans. Evol. Comput. 2003, 7, 83-94. [CrossRef]

26. Yuan, D.M.; Ho, D.W.C. Randomized gradient-free method for multi agent optimization over time-varying networks. IEEE Trans. Neural Learn. 2015, 26, 1342-1347. [CrossRef] [PubMed] 